\title{
Variable Chains Found in Mixed Transition Metal Oxyfluorides with Heterocyclic Ligands
}

\author{
Belal Ahmed, ${ }^{a}$ Hongil Jo, ${ }^{a}$ and Kang Min Ok ${ }^{b, *}$ \\ aDepartment of Chemistry, Chung-Ang University, Seoul 06974, Republic of Korea \\ bDepartment of Chemistry, Sogang University, Seoul 04107, Republic of Korea \\ E-mail: kmok@sogang.ac.kr
}

\section{Contents}

Figure S1. Experimental and calculated powder X-ray diffraction patterns for compound 1 Figure S2. Experimental and calculated powder X-ray diffraction patterns for compound 2 Figure S3. Experimental and calculated powder X-ray diffraction patterns for compound 3 Figure S4. Experimental and calculated powder X-ray diffraction patterns for compound 4 Figure S5. Experimental and calculated powder X-ray diffraction patterns for compound 5 Figure S6. Experimental and calculated powder X-ray diffraction patterns for compound 6 Figure S7. EDX spectrum for compound 1

Figure S8. EDX spectrum for compound 2

Figure S9. EDX spectrum for compound 3

Figure S10. EDX spectrum for compound 4

Figure S11. EDX spectrum for compound 5

Figure S12. EDX spectrum for compound 6

Figure S13. IR spectra for compounds $1-6$

Figure S14. UV-vis diffuse reflectance spectrum of compound 1

Figure S15. UV-vis diffuse reflectance spectrum of compound 2

Figure S16. UV-vis diffuse reflectance spectrum of compound 3

Figure S17. UV-vis diffuse reflectance spectrum of compound 4

Figure S18. UV-vis diffuse reflectance spectrum of compound 5

Figure S19. UV-vis diffuse reflectance spectrum of compound 6

Figure S20. TGA diagrams for compounds 1-6

Figure S21. Powder X-ray diffraction patterns of compound 1 at different temperatures Figure S22. Powder X-ray diffraction patterns of compound 2 at different temperatures Figure S23. Powder X-ray diffraction patterns of compound 3 at different temperatures Figure S24. Powder X-ray diffraction patterns of compound 4 at different temperatures Figure S25. Powder X-ray diffraction patterns of compound 5 at different temperatures Figure S26. Powder X-ray diffraction patterns of compound 6 at different temperatures 
Table S1. Selected bond distances $(\AA)$ and bond angles (degree) for compound 1

Table S2. Selected bond distances $(\AA)$ and bond angles (degree) for compound 2

Table S3. Selected bond distances $(\AA)$ and bond angles (degree) for compound 3

Table S4. Selected bond distances $(\AA)$ and bond angles (degree) for compound 4

Table S5. Selected bond distances $(\AA)$ and bond angles (degree) for compound 5

Table S6. Selected bond distances $(\AA)$ and bond angles (degree) for compound 6

Table S7. EDX results for compounds 1-6

Table S8. Bond valence sum for compound 1

Table S9. Bond valence sum for compound 2

Table S10. Bond valence sum for compound 3

Table S11. Bond valence sum for compound 4

Table S12. Bond valence sum for compound 5

Table S13. Bond valence sum for compound 6 
Figure S1. Experimental and calculated powder X-ray diffraction patterns for compound 1

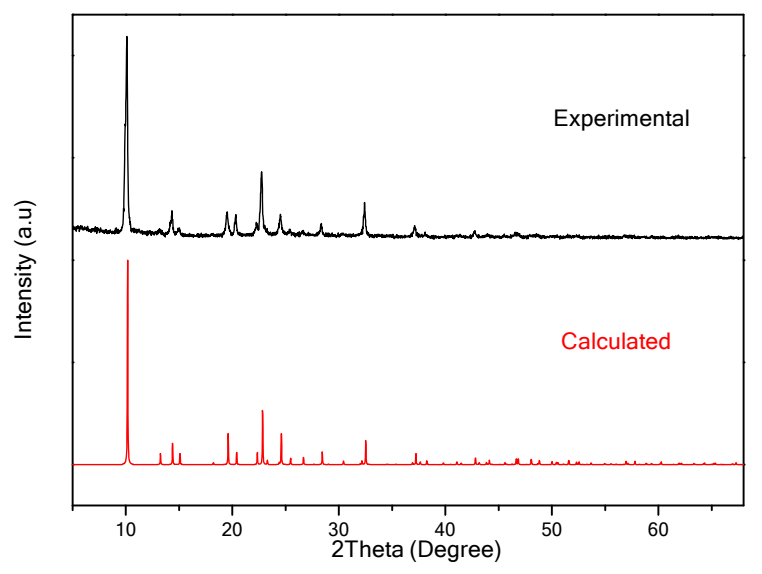

Figure S2. Experimental and calculated powder X-ray diffraction patterns for compound 2

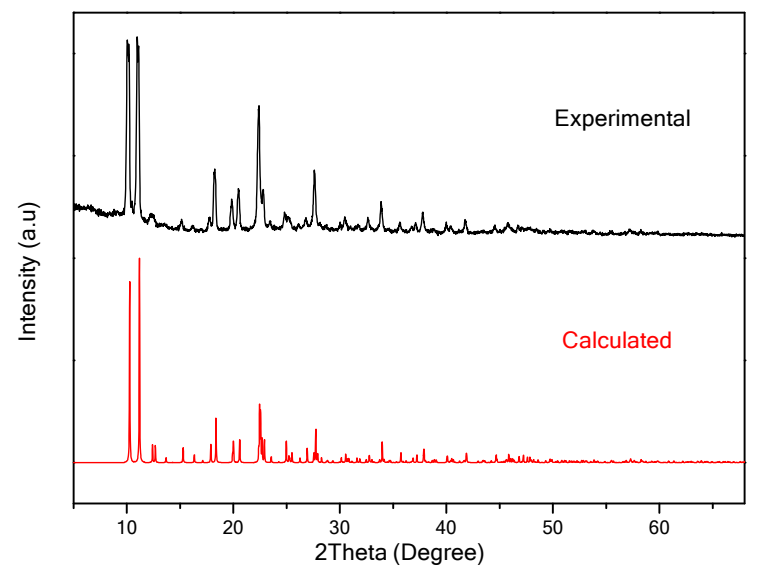

Figure S3. Experimental and calculated powder X-ray diffraction patterns for compound 3 


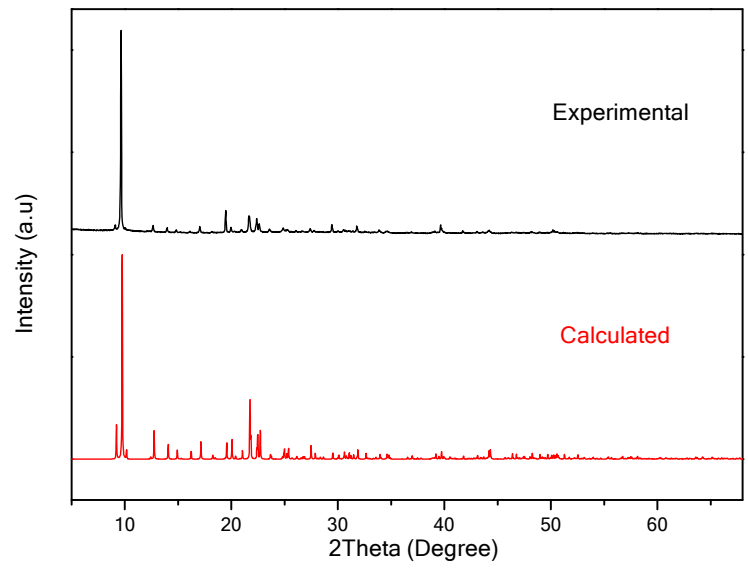

Figure S4. Experimental and calculated powder X-ray diffraction patterns for compound 4

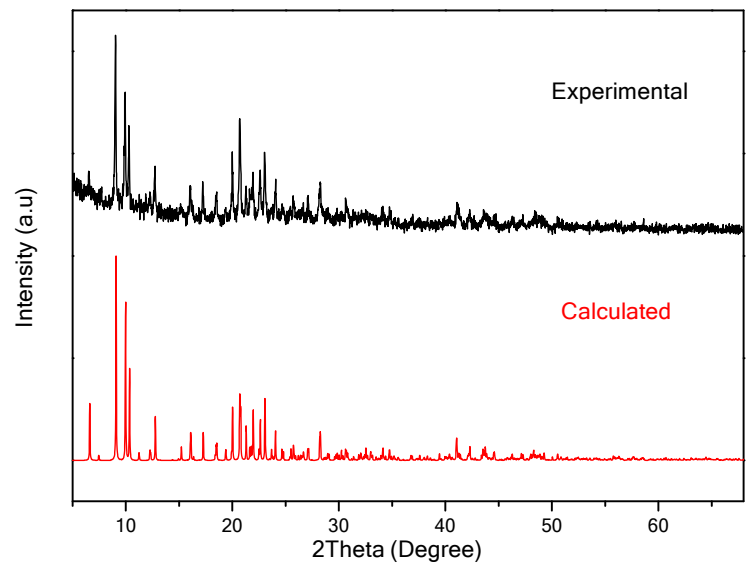


Figure S5. Experimental and calculated powder X-ray diffraction patterns for compound 5

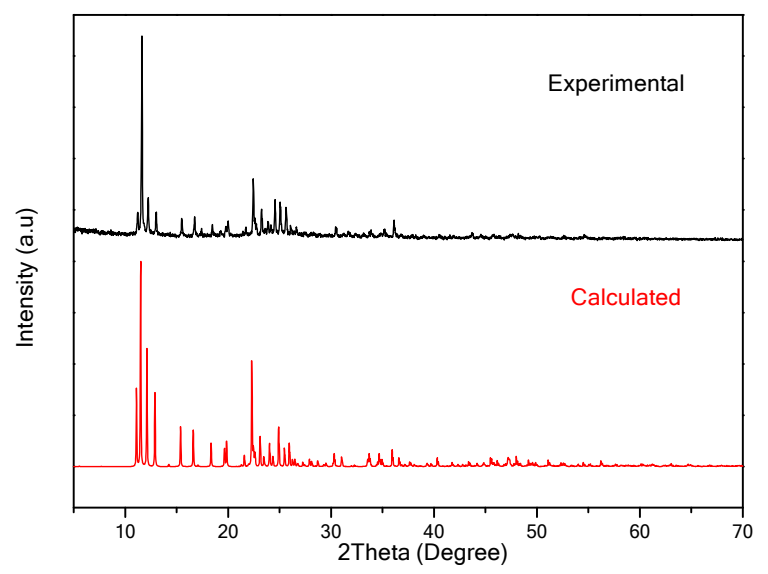

Figure S6. Experimental and calculated powder X-ray diffraction patterns for compound 6

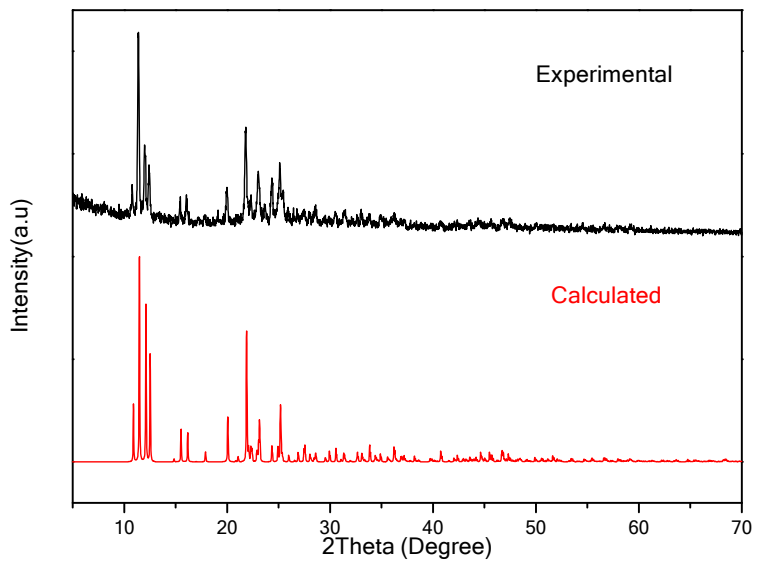


Figure S7. EDX spectrum for compound 1

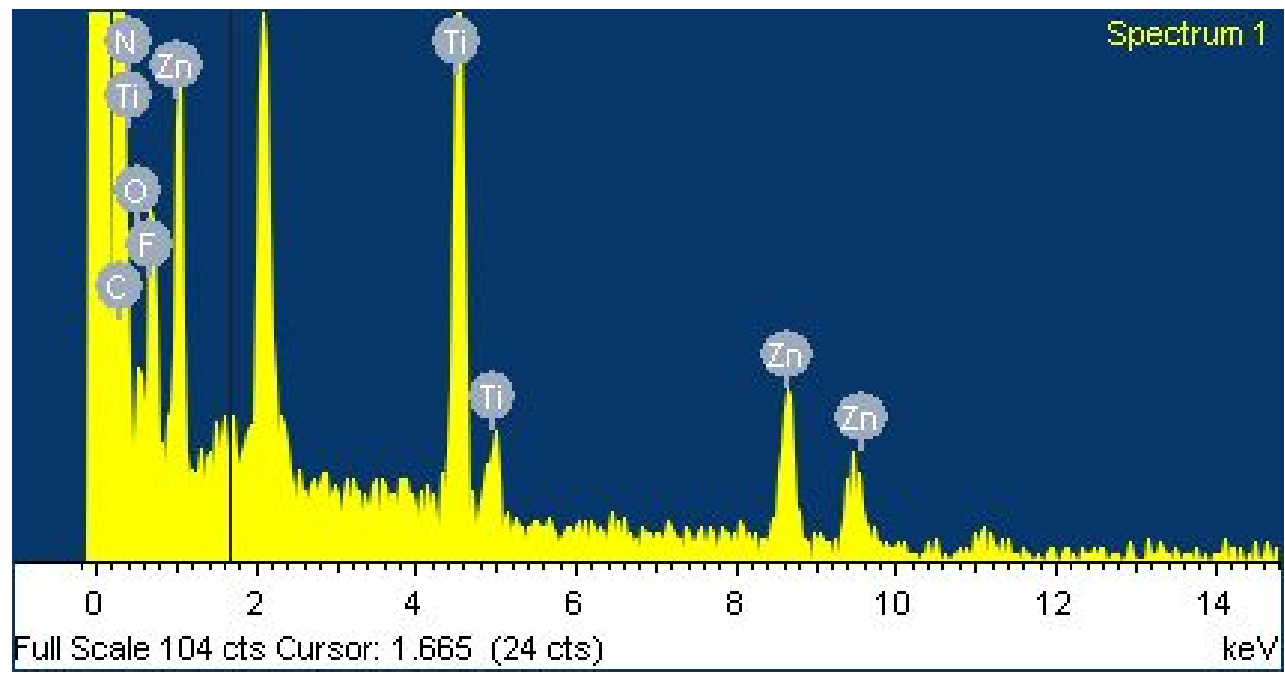

Figure S8. EDX spectrum for compound 2

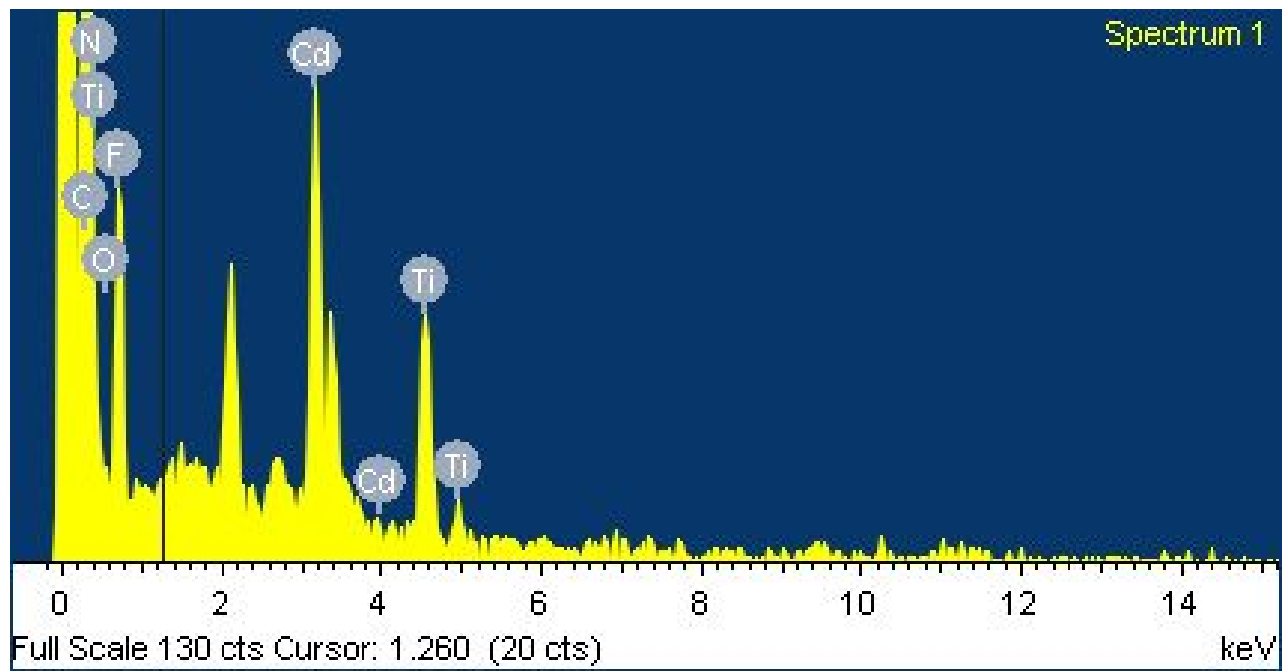

Figure S9. EDX spectrum for compound 3

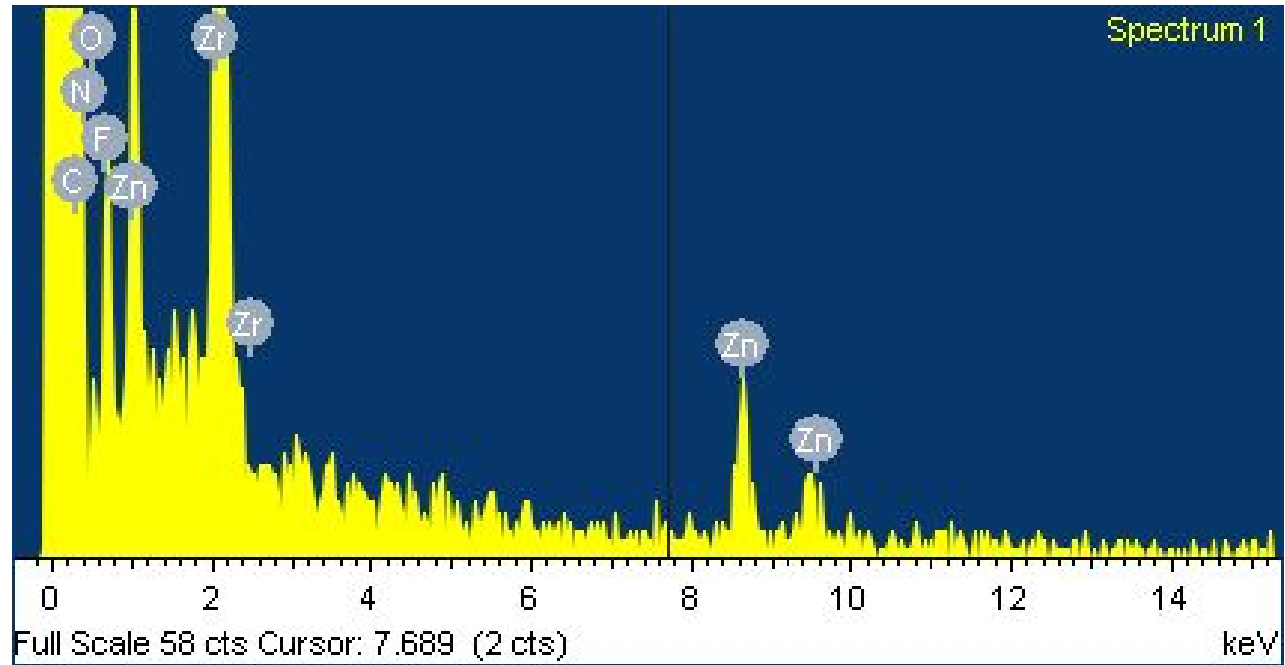


Figure S10. EDX spectrum for compound 4

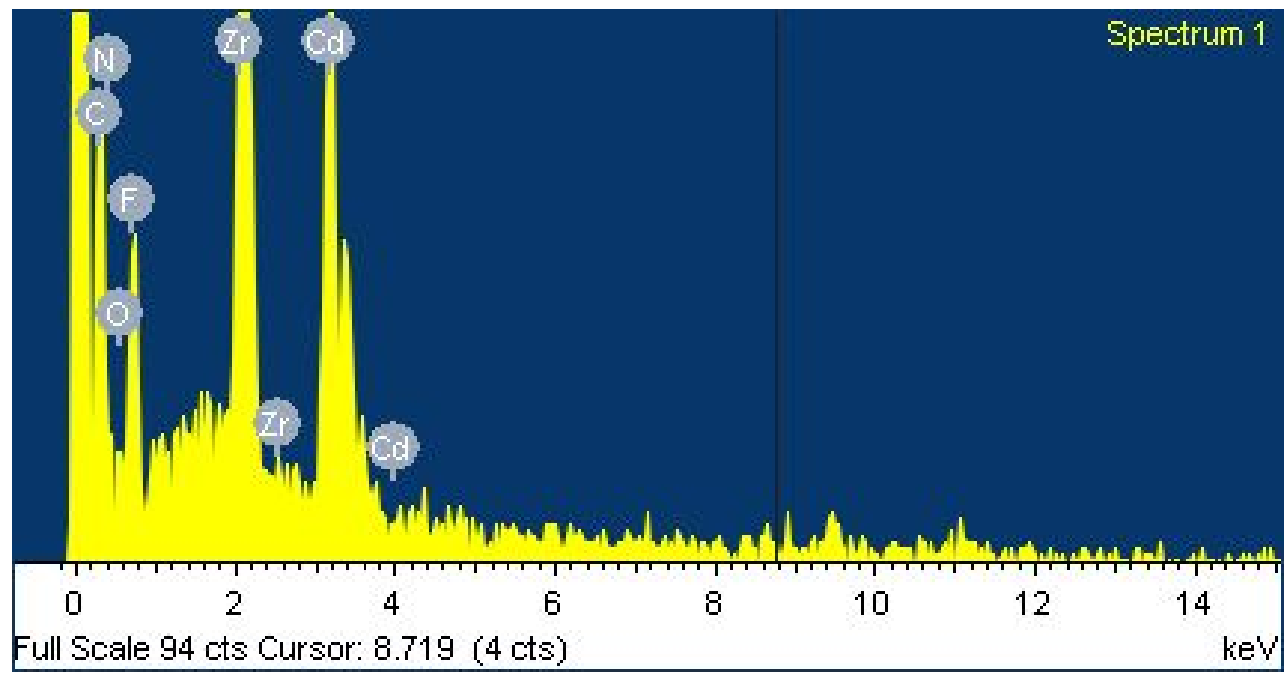

Figure S11. EDX spectrum for compound 5

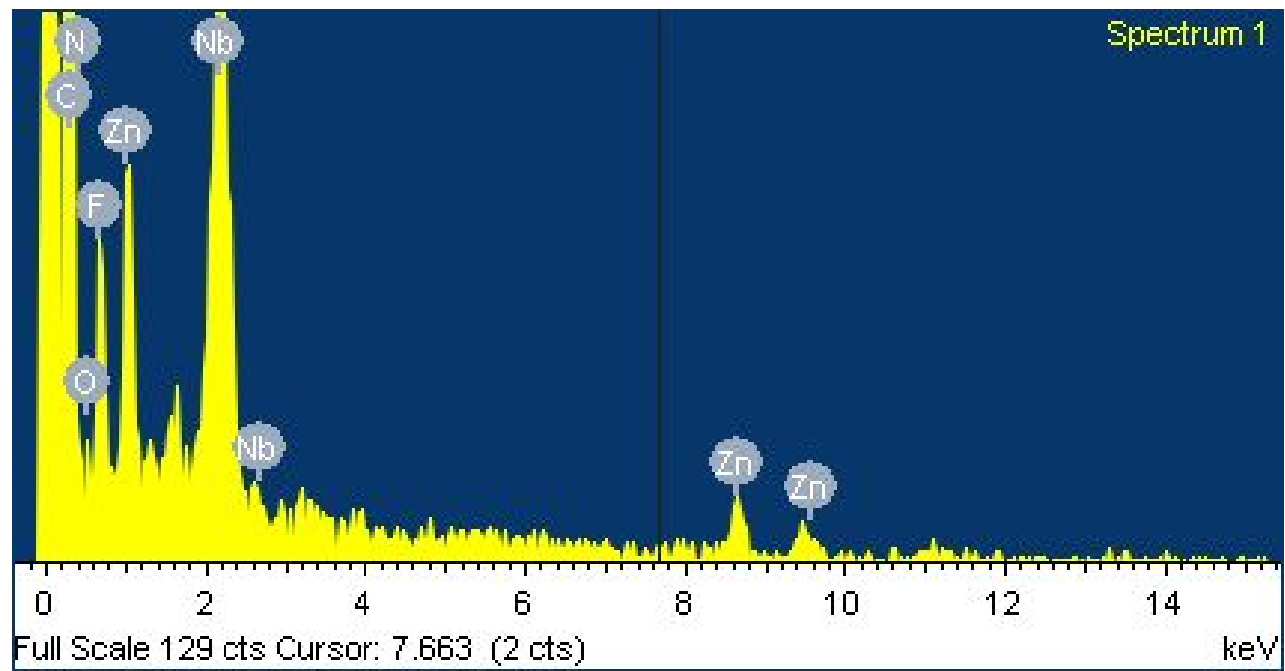

Figure S12. EDX spectrum for compound 6

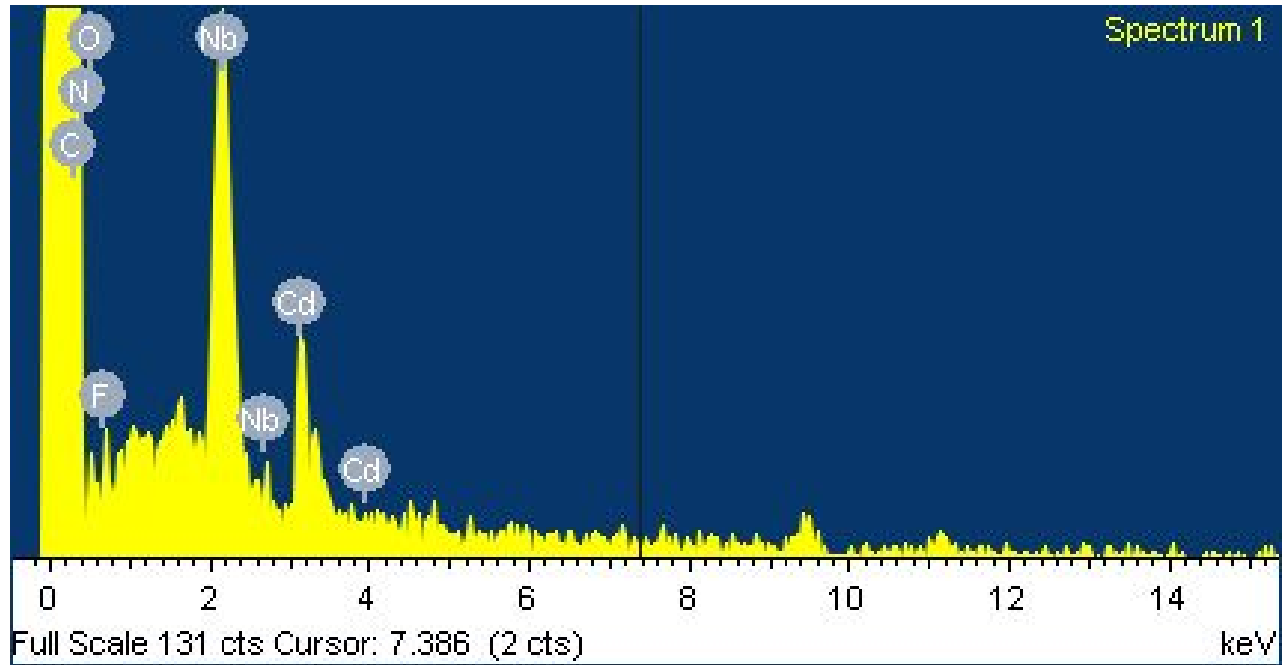


Figure S13. IR spectra for compounds 1-6

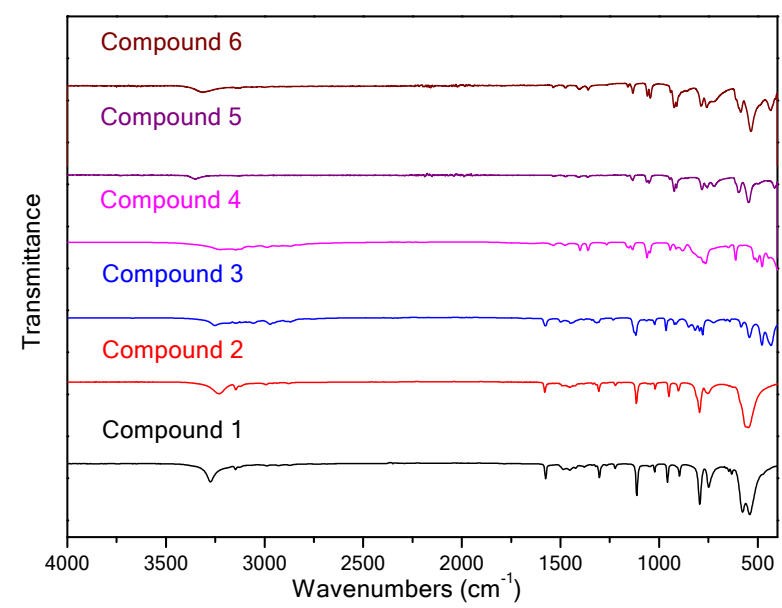

Figure S14. UV-vis diffuse reflectance spectrum of compound 1

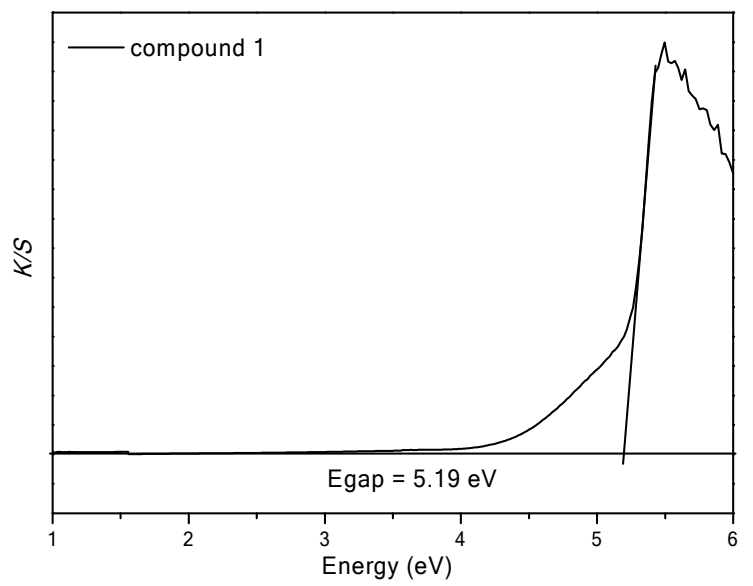


Figure S15. UV-vis diffuse reflectance spectrum of compound 2

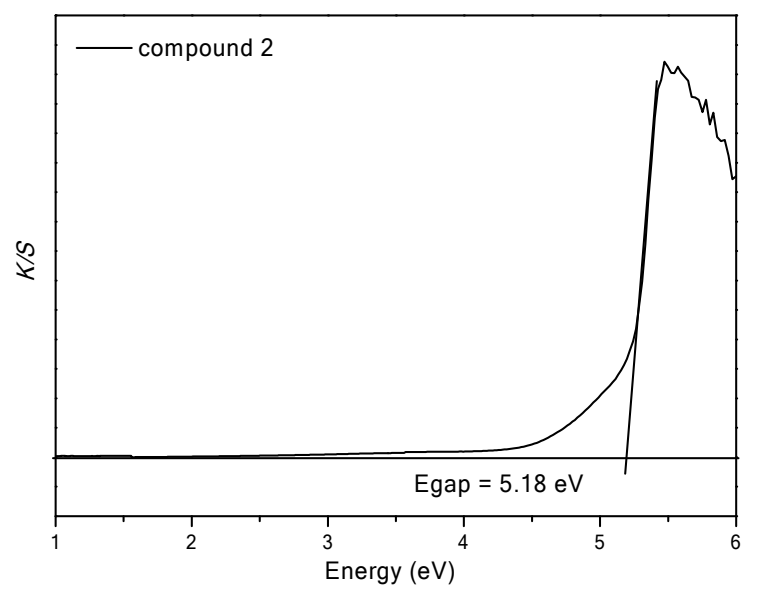

Figure S16. UV-vis diffuse reflectance spectrum of compound 3

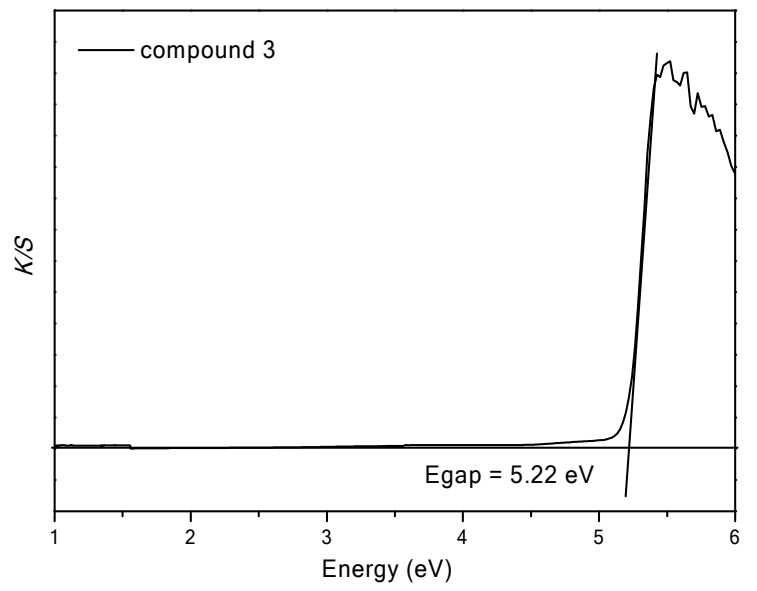


Figure S17. UV-vis diffuse reflectance spectrum of compound 4

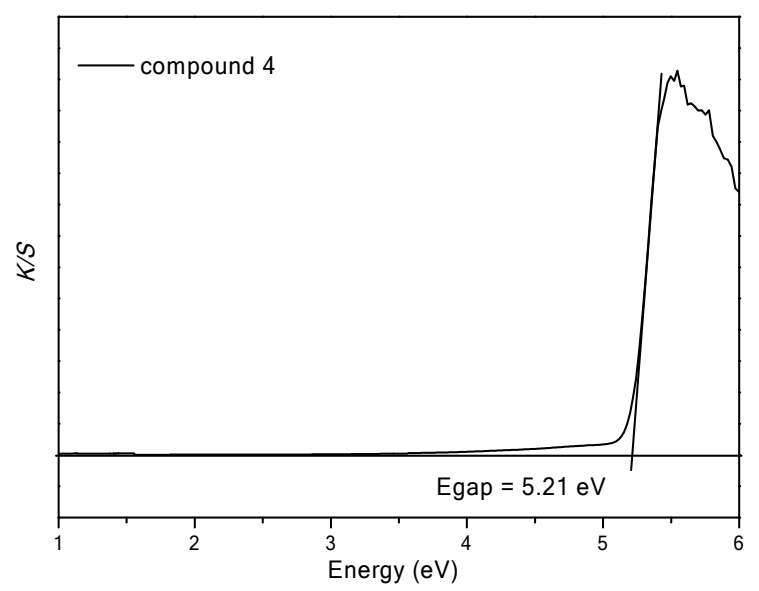

Figure S18. UV-vis diffuse reflectance spectrum of compound 5

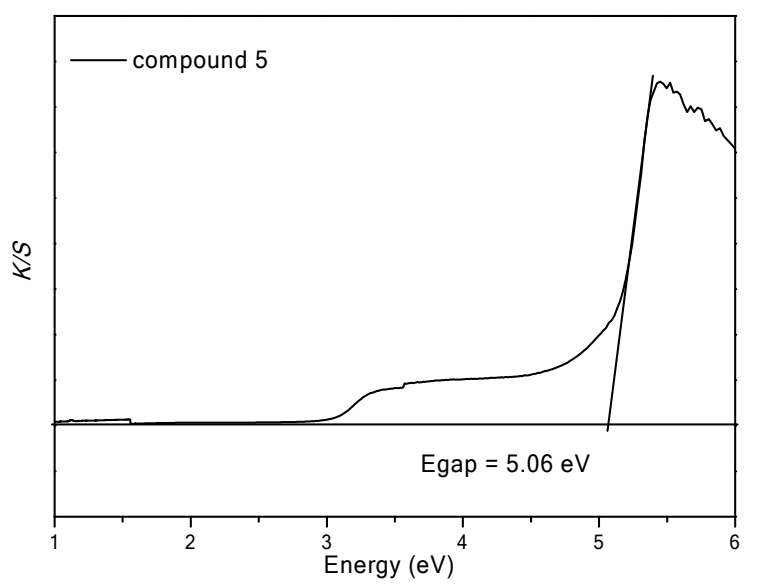


Figure S19. UV-vis diffuse reflectance spectrum of compound 6

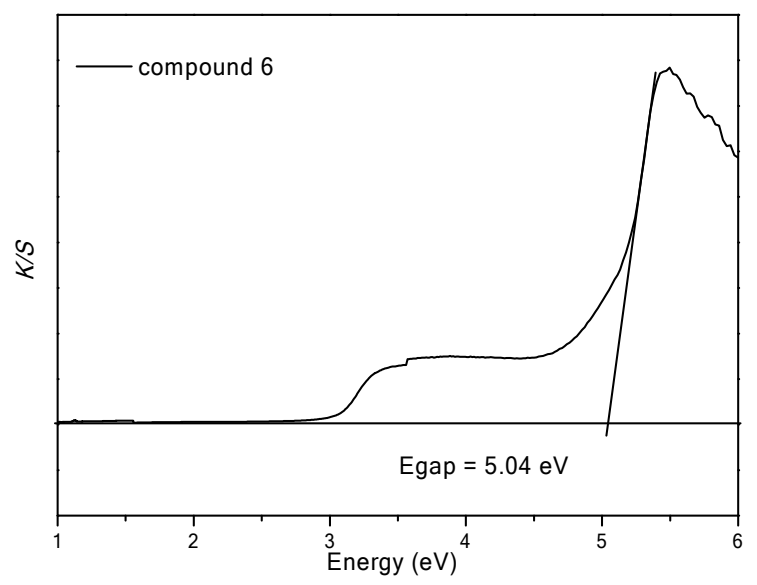

Figure S20. TGA diagrams for compounds 1-6

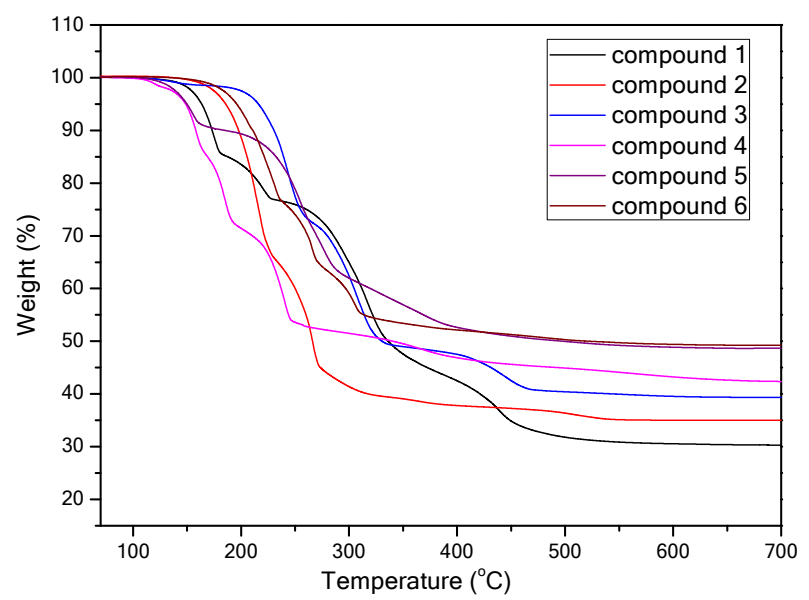


Figure S21. Powder X-ray diffraction patterns of compound 1 at different temperatures

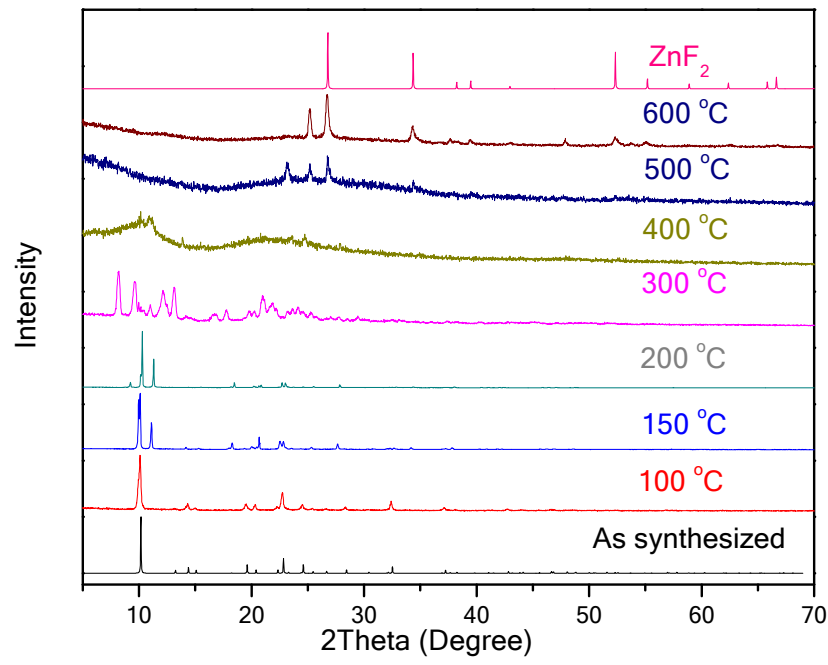

Figure S22. Powder X-ray diffraction patterns of compound 2 at different temperatures

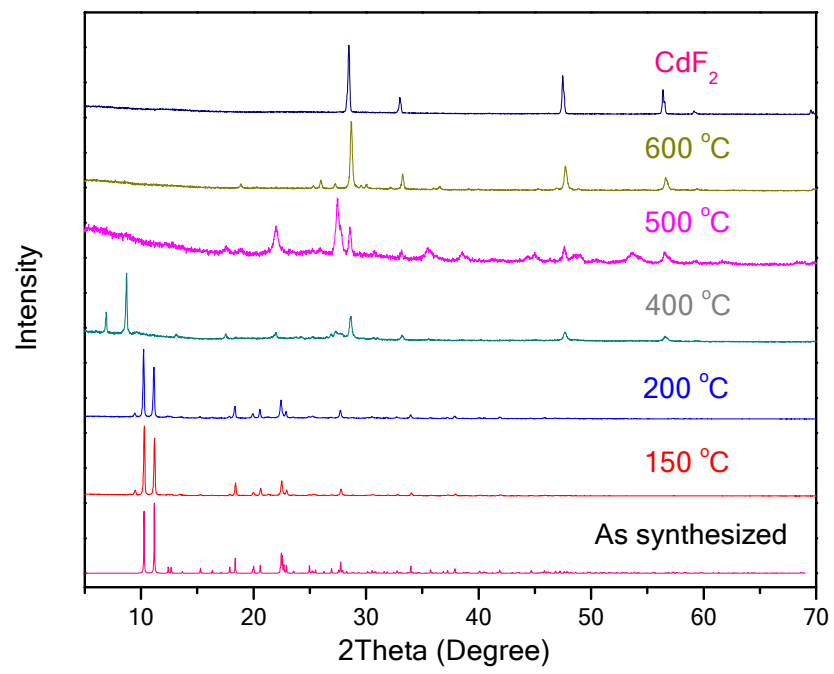


Figure S23. Powder X-ray diffraction patterns of compound 3 at different temperatures

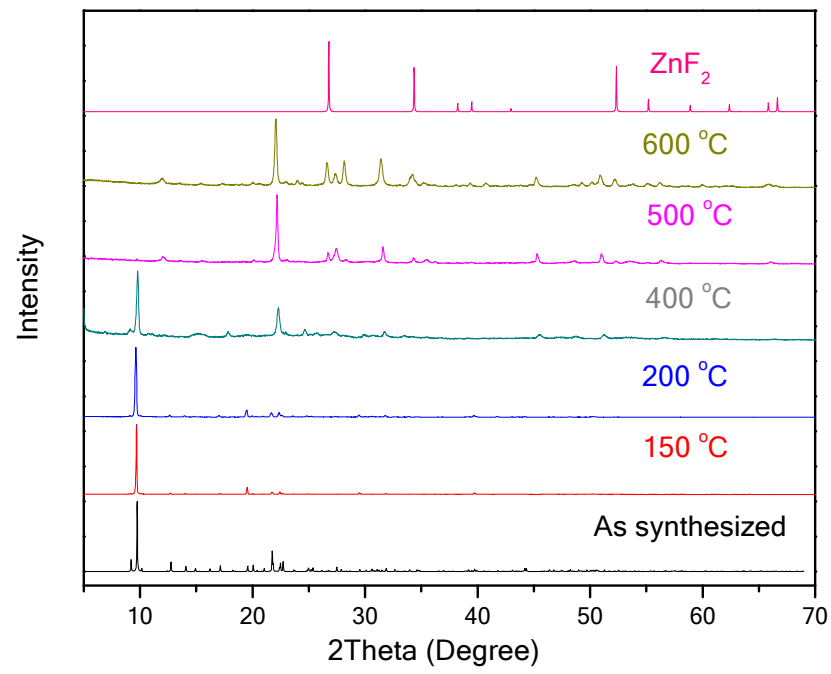

Figure S24. Powder X-ray diffraction patterns of compound 4 at different temperatures

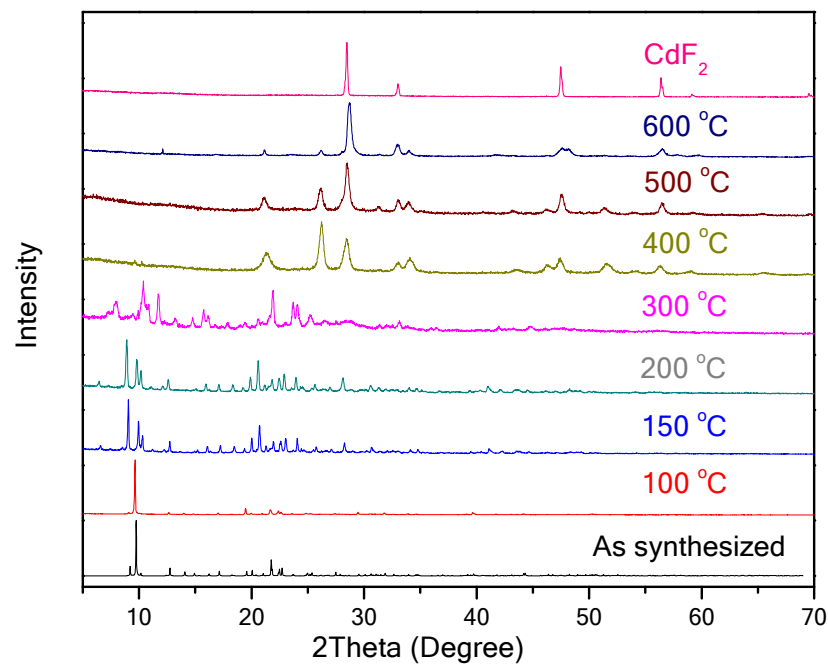


Figure S25. Powder X-ray diffraction patterns of compound 5 at different temperatures

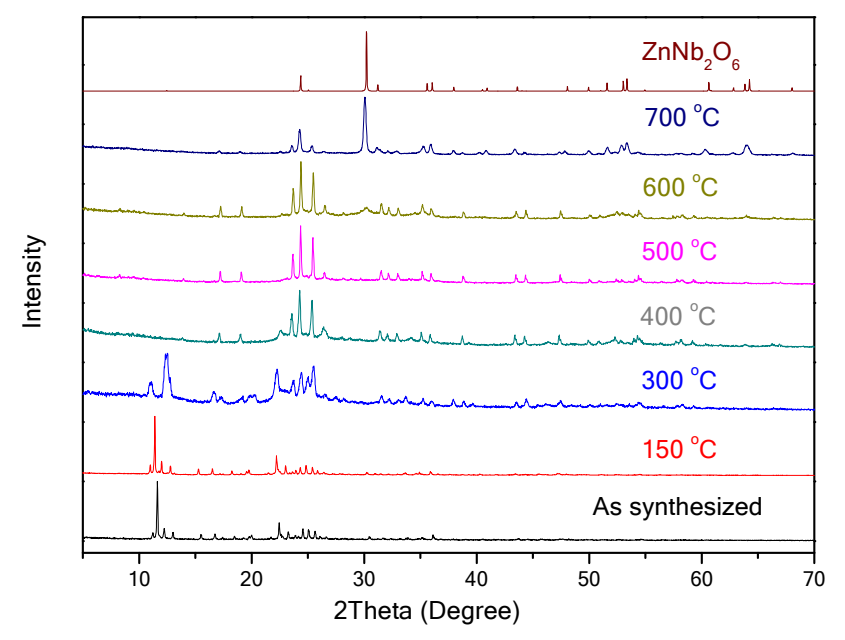

Figure S26. Powder X-ray diffraction patterns of compound 6 at different temperatures

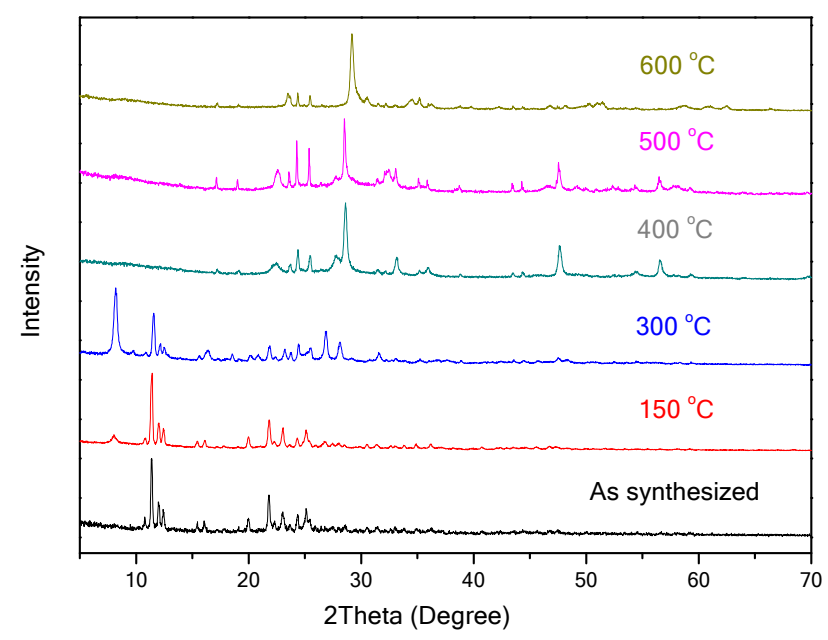


Table S1. Selected bond distances $(\AA)$ and bond angles (degree) for compound 1

\begin{tabular}{cccc}
\hline $\mathrm{Zn}(1)-\mathrm{N}(1)$ & $2.1216(14)$ & $\mathrm{Ti}(1)-\mathrm{F}(1)$ & $1.8821(15)$ \\
$\mathrm{Zn}(1)-\mathrm{F}(1)$ & $2.0939(15)$ & $\mathrm{Ti}(1)-\mathrm{F}(2)$ & $1.8439(12)$ \\
\hline & & & \\
\hline $\mathrm{F}(1)-\mathrm{Ti}(1)-\mathrm{F}(1)$ & 180 & $\mathrm{~F}(2)-\mathrm{Ti}(1)-\mathrm{F}(2)$ & $174.24(5)$ \\
$\mathrm{F}(1)-\mathrm{Ti}(1)-\mathrm{F}(2)$ & $87.12(2)$ & $\mathrm{F}(2)-\mathrm{Ti}(1)-\mathrm{F}(2)$ & $90.146(3)$ \\
$\mathrm{F}(1)-\mathrm{Ti}(1)-\mathrm{F}(2)$ & $92.88(2)$ & $\mathrm{F}(2)-\mathrm{Ti}(1)-\mathrm{F}(2)$ & $90.144(3)$ \\
$\mathrm{F}(1)-\mathrm{Zn}(1)-\mathrm{F}(1)$ & 180 & & \\
\hline
\end{tabular}

Table S2. Selected bond distances $(\AA)$ and bond angles (degree) for compound 2

\begin{tabular}{cccc}
\hline $\mathrm{Cd}(1)-\mathrm{N}(1)$ & $2.2799(13)$ & $\mathrm{Ti}(1)-\mathrm{F}(1)$ & $1.8632(15)$ \\
$\mathrm{Cd}(1)-\mathrm{N}(3)$ & $2.3156(13)$ & $\mathrm{Ti}(1)-\mathrm{F}(2)$ & $1.8444(11)$ \\
$\mathrm{Cd}(1)-\mathrm{F}(1)$ & $2.2698(17)$ & $\mathrm{Ti}(1)-\mathrm{F}(3)$ & $1.8448(11)$ \\
\hline & & & \\
\hline $\mathrm{F}(1)-\mathrm{Ti}(1)-\mathrm{F}(1)$ & $180.00(11)$ & $\mathrm{F}(2)-\mathrm{Ti}(1)-\mathrm{F}(2)$ & 180 \\
$\mathrm{~F}(1)-\mathrm{Ti}(1)-\mathrm{F}(2)$ & $90.57(7)$ & $\mathrm{F}(2)-\mathrm{Ti}(1)-\mathrm{F}(3)$ & $90.44(6)$ \\
$\mathrm{F}(1)-\mathrm{Ti}(1)-\mathrm{F}(2)$ & $89.44(7)$ & $\mathrm{F}(2)-\mathrm{Ti}(1)-\mathrm{F}(3)$ & $89.55(6)$ \\
$\mathrm{F}(1)-\mathrm{Ti}(1)-\mathrm{F}(3)$ & $90.55(7)$ & $\mathrm{F}(3)-\mathrm{Ti}(1)-\mathrm{F}(3)$ & 180 \\
$\mathrm{~F}(1)-\mathrm{Ti}(1)-\mathrm{F}(3)$ & $89.45(7)$ & $\mathrm{F}(1)-\mathrm{Cd}(1)-\mathrm{F}(1)$ & 180 \\
\hline
\end{tabular}

Table S3. Selected bond distances $(\AA)$ and bond angles (degree) for compound 3

\begin{tabular}{llll}
\hline $\mathrm{Zn}(1)-\mathrm{N}(1)$ & $2.006(4)$ & $\mathrm{Zr}(1)-\mathrm{F}(1)$ & $2.094(2)$ \\
$\mathrm{Zn}(1)-\mathrm{N}(3)$ & $2.007(4)$ & $\mathrm{Zr}(1)-\mathrm{F}(2)$ & $1.946(3)$ \\
$\mathrm{Zn}(1)-\mathrm{N}(5)$ & $1.998(4)$ & $\mathrm{Zr}(1)-\mathrm{F}(3)$ & $1.999(3)$ \\
$\mathrm{Zn}(1)-\mathrm{F}(1)$ & $2.163(2)$ & $\mathrm{Zr}(2)-\mathrm{F}(4)$ & $2.003(3)$ \\
$\mathrm{Zn}(1)-\mathrm{F}(6)$ & $2.136(2)$ & $\mathrm{Zr}(2)-\mathrm{F}(5)$ & $2.170(2)$ \\
$\mathrm{Zr}(2)-\mathrm{F}(6)$ & $2.069(2)$ & $\mathrm{Zr}(2)-\mathrm{F}(5)$ & \\
\hline
\end{tabular}




\begin{tabular}{|c|c|c|c|}
\hline $\mathrm{F}(1)-\mathrm{Zr}(1)-\mathrm{F}(2)$ & $92.92(13)$ & $\mathrm{F}(3)-\mathrm{Zr}(1)-\mathrm{F}(4)$ & $87.17(12)$ \\
\hline $\mathrm{F}(1)-\mathrm{Zr}(1)-\mathrm{F}(3)$ & $150.61(11)$ & $F(3)-\operatorname{Zr}(1)-F(5)$ & $72.41(10)$ \\
\hline $\mathrm{F}(1)-\mathrm{Zr}(1)-\mathrm{F}(4)$ & $82.64(11)$ & $\mathrm{F}(3)-\mathrm{Zr}(1)-\mathrm{F}(5)$ & $135.80(10)$ \\
\hline $\mathrm{F}(1)-\mathrm{Zr}(1)-\mathrm{F}(5)$ & $71.37(9)$ & $\mathrm{F}(3)-\mathrm{Zr}(1)-\mathrm{F}(6)$ & $76.33(11)$ \\
\hline $\mathrm{F}(1)-\mathrm{Zr}(1)-\mathrm{F}(5)$ & $134.74(9)$ & $\mathrm{F}(4)-\mathrm{Zr}(1)-\mathrm{F}(5)$ & $87.56(11)$ \\
\hline $\mathrm{F}(1)-\mathrm{Zr}(1)-\mathrm{F}(6)$ & $76.71(10)$ & $\mathrm{F}(4)-\mathrm{Zr}(1)-\mathrm{F}(5)$ & $89.92(12)$ \\
\hline $\mathrm{F}(2)-\mathrm{Zr}(1)-\mathrm{F}(3)$ & $98.16(15)$ & $\mathrm{F}(4)-\mathrm{Zr}(1)-\mathrm{F}(6)$ & $92.71(12)$ \\
\hline $\mathrm{F}(2)-\mathrm{Zr}(1)-\mathrm{F}(4)$ & $174.63(13)$ & $\mathrm{F}(5)-\mathrm{Zr}(1)-\mathrm{F}(5)$ & $63.73(11)$ \\
\hline $\mathrm{F}(2)-\mathrm{Zr}(1)-\mathrm{F}(5)$ & $88.15(12)$ & $\mathrm{F}(5)-\mathrm{Zr}(1)-\mathrm{F}(6)$ & $147.78(10)$ \\
\hline $\mathrm{F}(2)-\mathrm{Zr}(1)-\mathrm{F}(5)$ & $91.08(13)$ & $\mathrm{F}(5)-\mathrm{Zr}(1)-\mathrm{F}(6)$ & $148.45(9)$ \\
\hline $\mathrm{F}(2)-\mathrm{Zr}(1)-\mathrm{F}(6)$ & $89.20(12)$ & $\mathrm{F}(1)-\mathrm{Zn}(1)-\mathrm{F}(6)$ & $177.10(10)$ \\
\hline
\end{tabular}

Table S4. Selected bond distances $(\AA)$ and bond angles (degree) for compound 4

\begin{tabular}{cccc}
\hline $\mathrm{Cd}(1)-\mathrm{N}(1)$ & $2.304(3)$ & $\mathrm{Zr}(1)-\mathrm{F}(1)$ & $2.040(2)$ \\
$\mathrm{Cd}(1)-\mathrm{N}(3)$ & $2.280(3)$ & $\mathrm{Zr}(1)-\mathrm{F}(2)$ & $2.011(2)$ \\
$\mathrm{Cd}(1)-\mathrm{N}(5)$ & $2.303(3)$ & $\mathrm{Zr}(1)-\mathrm{F}(3)$ & $1.971(2)$ \\
$\mathrm{Cd}(1)-\mathrm{N}(7)$ & $2.316(3)$ & $\mathrm{Zr}(2)-\mathrm{F}(4)$ & $2.112(2)$ \\
$\mathrm{Cd}(1)-\mathrm{F}(1)$ & $2.397(2)$ & $\mathrm{Zr}(2)-\mathrm{F}(5)$ & $2.104(19)$ \\
$\mathrm{Cd}(1)-\mathrm{F}(4)$ & $2.354(2)$ & $\mathrm{Zr}(2)-\mathrm{F}(6)$ & $2.136(2)$ \\
$\mathrm{Cd}(2)-\mathrm{N}(9)$ & $2.325(3)$ & $\mathrm{Zr}(2)-\mathrm{F}(7)$ & $2.130(2)$ \\
$\mathrm{Cd}(2)-\mathrm{N}(11)$ & $2.267(3)$ & $\mathrm{Cd}(2)-\mathrm{F}(5)$ & $2.3526(18)$ \\
\hline $\mathrm{F}(1)-\mathrm{Zr}(1)-\mathrm{F}(1)$ & $85.27(13)$ & $\mathrm{F}(2)-\mathrm{Zr}(1)-\mathrm{F}(2)$ & $170.37(13)$ \\
$\mathrm{F}(1)-\mathrm{Zr}(1)-\mathrm{F}(2)$ & $89.05(9)$ & $\mathrm{F}(3)-\mathrm{Zr}(1)-\mathrm{F}(3)$ & $93.24(15)$ \\
$\mathrm{F}(1)-\mathrm{Zr}(1)-\mathrm{F}(2)$ & $83.87(9)$ & $\mathrm{F}(1)-\mathrm{Cd}(1)-\mathrm{F}(4)$ & $171.47(8)$ \\
$\mathrm{F}(1)-\mathrm{Zr}(1)-\mathrm{F}(3)$ & $90.83(10)$ & $\mathrm{F}(5)-\mathrm{Zr}(2)-\mathrm{F}(5)$ & $142.54(12)$ \\
$\mathrm{F}(1)-\mathrm{Zr}(1)-\mathrm{F}(3)$ & $174.91(11)$ & $\mathrm{F}(5)-\mathrm{Zr}(2)-\mathrm{F}(6)$ & $74.08(8)$ \\
\hline
\end{tabular}




\begin{tabular}{lclc}
\hline $\mathrm{F}(2)-\operatorname{Zr}(1)-\mathrm{F}(3)$ & $92.80(11)$ & $\mathrm{F}(5)-\mathrm{Zr}(2)-\mathrm{F}(6)$ & $77.02(8)$ \\
$\mathrm{F}(2)-\operatorname{Zr}(1)-\mathrm{F}(3)$ & $93.81(11)$ & $\mathrm{F}(5)-\operatorname{Zr}(2)-\mathrm{F}(7)$ & $72.11(9)$ \\
$\mathrm{F}(4)-\operatorname{Zr}(2)-\mathrm{F}(4)$ & $139.50(13)$ & $\mathrm{F}(5)-\operatorname{Zr}(2)-\mathrm{F}(7)$ & $144.49(9)$ \\
$\mathrm{F}(4)-\operatorname{Zr}(2)-\mathrm{F}(5)$ & $107.82(9)$ & $\mathrm{F}(6)-\mathrm{Zr}(2)-\mathrm{F}(6)$ & $78.05(11)$ \\
$\mathrm{F}(4)-\operatorname{Zr}(2)-\mathrm{F}(5)$ & $85.19(9)$ & $\mathrm{F}(6)-\operatorname{Zr}(2)-\mathrm{F}(7)$ & $137.03(9)$ \\
$\mathrm{F}(4)-\operatorname{Zr}(2)-\mathrm{F}(6)$ & $147.07(9)$ & $\mathrm{F}(6)-\operatorname{Zr}(2)-\mathrm{F}(7)$ & $119.63(9)$ \\
$\mathrm{F}(4)-\operatorname{Zr}(2)-\mathrm{F}(6)$ & $72.45(9)$ & $\mathrm{F}(7)-\operatorname{Zr}(2)-\mathrm{F}(7)$ & $75.79(13)$ \\
$\mathrm{F}(4)-\operatorname{Zr}(2)-\mathrm{F}(7)$ & $72.46(10)$ & $\mathrm{F}(5)-\mathrm{Cd}(2)-\mathrm{F}(5)$ & 180 \\
$\mathrm{~F}(4)-\operatorname{Zr}(2)-\mathrm{F}(7)$ & $75.82(9)$ & & \\
\hline
\end{tabular}

Table S5. Selected bond distances $(\AA)$ and bond angles (degree) for compound 5

\begin{tabular}{cccc}
\hline $\mathrm{Zn}(1)-\mathrm{N}(1)$ & $2.121(2)$ & $\mathrm{Nb}(1)-\mathrm{O}(1)$ & $1.7581(17)$ \\
$\mathrm{Zn}(1)-\mathrm{N}(3)$ & $2.162(9)$ & $\mathrm{Nb}(1)-\mathrm{F}(1)$ & $2.1031(14)$ \\
$\mathrm{Zn}(1)-\mathrm{N}(5)$ & $2.152(2)$ & $\mathrm{Nb}(1)-\mathrm{F}(2)$ & $1.9313(18)$ \\
$\mathrm{Zn}(1)-\mathrm{N}(7)$ & $2.123(2)$ & $\mathrm{Nb}(1)-\mathrm{F}(3)$ & $1.9233(16)$ \\
$\mathrm{Zn}(1)-\mathrm{O}(1)$ & $2.1093(17)$ & $\mathrm{Nb}(1)-\mathrm{F}(4)$ & $1.9267(17)$ \\
$\mathrm{Zn}(1)-\mathrm{F}(6)$ & $2.1447(14)$ & $\mathrm{Nb}(1)-\mathrm{F}(5)$ & $1.9290(18)$ \\
$\mathrm{Zn}(2)-\mathrm{N}(9)$ & $2.124(2)$ & $\mathrm{Nb}(2)-\mathrm{O}(2)$ & $1.7529(17)$ \\
$\mathrm{Zn}(2)-\mathrm{N}(11)$ & $2.117(2)$ & $\mathrm{Nb}(2)-\mathrm{F}(6)$ & $2.1069(14)$ \\
$\mathrm{Zn}(2)-\mathrm{N}(13)$ & $2.158(2)$ & $\mathrm{Nb}(2)-\mathrm{F}(7)$ & $1.9247(16)$ \\
$\mathrm{Zn}(2)-\mathrm{N}(15)$ & $2.157(2)$ & $\mathrm{Nb}(2)-\mathrm{F}(8)$ & $1.9442(17)$ \\
$\mathrm{Zn}(2)-\mathrm{O}(2)$ & $2.1248(17)$ & $\mathrm{Nb}(2)-\mathrm{F}(9)$ & $1.9250(18)$ \\
$\mathrm{Zn}(2)-\mathrm{F}(1)$ & $2.1396(14)$ & $\mathrm{Nb}(2)-\mathrm{F}(10)$ & $1.9247(17)$ \\
\hline
\end{tabular}

\begin{tabular}{cccc}
\hline $\mathrm{O}(1)-\mathrm{Nb}(1)-\mathrm{F}(1)$ & $178.20(7)$ & $\mathrm{O}(2)-\mathrm{Nb}(2)-\mathrm{F}(6)$ & $179.06(7)$ \\
$\mathrm{O}(1)-\mathrm{Nb}(1)-\mathrm{F}(2)$ & $96.05(8)$ & $\mathrm{O}(2)-\mathrm{Nb}(2)-\mathrm{F}(7)$ & $97.37(8)$ \\
\hline
\end{tabular}




\begin{tabular}{lccc}
\hline $\mathrm{O}(1)-\mathrm{Nb}(1)-\mathrm{F}(3)$ & $97.72(8)$ & $\mathrm{O}(2)-\mathrm{Nb}(2)-\mathrm{F}(8)$ & $94.79(8)$ \\
$\mathrm{O}(1)-\mathrm{Nb}(1)-\mathrm{F}(4)$ & $97.65(8)$ & $\mathrm{O}(2)-\mathrm{Nb}(2)-\mathrm{F}(9)$ & $97.32(8)$ \\
$\mathrm{O}(1)-\mathrm{Nb}(1)-\mathrm{F}(5)$ & $95.68(8)$ & $\mathrm{O}(2)-\mathrm{Nb}(2)-\mathrm{F}(10)$ & $97.86(8)$ \\
$\mathrm{O}(1)-\mathrm{Zn}(1)-\mathrm{F}(6)$ & $179.31(8)$ & $\mathrm{O}(2)-\mathrm{Zn}(2)-\mathrm{F}(1)$ & $179.48(8)$ \\
\hline
\end{tabular}

Table S6. Selected bond distances $(\AA)$ and bond angles (degree) for compound 6

\begin{tabular}{cccc}
\hline $\mathrm{Cd}(1)-\mathrm{N}(1)$ & $2.327(2)$ & $\mathrm{Nb}(1)-\mathrm{O}(1) / \mathrm{F}(1)$ & $1.9063(17)$ \\
$\mathrm{Cd}(1)-\mathrm{N}(3)$ & $2.310(2)$ & $\mathrm{Nb}(1)-\mathrm{F}(2)$ & $1.9180(19)$ \\
$\mathrm{Cd}(1)-\mathrm{O}(1) / \mathrm{F}(1)$ & $2.2555(17)$ & $\mathrm{Nb}(1)-\mathrm{F}(3)$ & $1.9216(19)$ \\
\hline & & & \\
\hline $\mathrm{O}(1) / \mathrm{F}(1)-\mathrm{Nb}(1)-\mathrm{F}(2)$ & $88.93(9)$ & $\mathrm{O}(1) / \mathrm{F}(1)-\mathrm{Nb}(1)-\mathrm{F}(3)$ & $90.85(9)$ \\
$\mathrm{O}(1) / \mathrm{F}(1)-\mathrm{Cd}(1)-\mathrm{O}(1) / \mathrm{F}(1)$ & 180 & & \\
\hline
\end{tabular}

Table S7. EDX results for compounds 1-6

\begin{tabular}{ccccccc}
\hline & Compound & Compound & Compound & Compound & Compound & Compound \\
& 1 & 2 & 3 & 4 & 4 & 5 \\
\hline $\mathrm{C} \mathrm{K}$ & 9.25 & 35.12 & 33.40 & 24.52 & 21.24 & 15.19 \\
$\mathrm{O} \mathrm{K}$ & -- & -- & -- & -- & 10.22 & 3.98 \\
$\mathrm{~N} \mathrm{~K}$ & 4.13 & -- & 25.19 & -- & -- & 7.97 \\
$\mathrm{~F} \mathrm{~K}$ & 6.87 & 15.44 & 10.25 & 8.27 & 27.33 & 8.90 \\
$\mathrm{Zn} \mathrm{L}$ & 1.44 & -- & 1.22 & -- & 1.00 & -- \\
$\mathrm{Cd} \mathrm{L}$ & -- & 1.34 & -- & 1.30 & -- & 1.00 \\
$\mathrm{Ti} \mathrm{K}$ & 1.00 & 1.00 & -- & -- & -- & -- \\
$\mathrm{Zr} \mathrm{L}$ & -- & -- & 1.00 & 1.00 & -- & -- \\
$\mathrm{Nb} \mathrm{L}$ & -- & -- & -- & -- & 1.94 & 1.12 \\
\hline
\end{tabular}


Table S8. Bond valence sum for compound 1

\begin{tabular}{cccccc}
\hline $\mathrm{Zn}(1)$ & 2.102 & $\mathrm{Ti}(1)$ & 3.934 & $\mathrm{~F}(1)$ & 0.889 \\
$\mathrm{~F}(2)$ & 0.678 & & & & \\
\hline
\end{tabular}

Table S9. Bond valence sum for compound 2

\begin{tabular}{llllll}
\hline $\mathrm{Cd}(1)$ & 2.186 & $\mathrm{Ti}(1)$ & 3.993 & $\mathrm{~F}(1)$ & 0.933 \\
$\mathrm{~F}(2)$ & 0.677 & $\mathrm{~F}(3)$ & 0.676 & & \\
\hline
\end{tabular}

Table S10. Bond valence sum for compound 3

\begin{tabular}{cccccc}
\hline $\mathrm{Zn}(1)$ & 2.074 & $\mathrm{Zr}(1)$ & 3.961 & $\mathrm{~F}(1)$ & 0.742 \\
$\mathrm{~F}(2)$ & 0.763 & $\mathrm{~F}(3)$ & 0.661 & $\mathrm{~F}(4)$ & 0.654 \\
$\mathrm{~F}(5)$ & 0.823 & $\mathrm{~F}(6)$ & 0.795 & & \\
\hline
\end{tabular}

Table S11. Bond valence sum for compound 4

\begin{tabular}{llllll}
\hline $\mathrm{Cd}(1)$ & 2.029 & $\mathrm{Cd}(2)$ & 2.081 & $\mathrm{Zr}(1)$ & 3.976 \\
$\mathrm{Zr}(2)$ & 3.895 & $\mathrm{~F}(1)$ & 0.810 & $\mathrm{~F}(2)$ & 0.654 \\
$\mathrm{~F}(3)$ & 0.729 & $\mathrm{~F}(4)$ & 0.728 & $\mathrm{~F}(5)$ & 0.740 \\
$\mathrm{~F}(6)$ & 0.467 & $\mathrm{~F}(7)$ & 0.474 & & \\
\hline
\end{tabular}

Table S12. Bond valence sum for compound 5

\begin{tabular}{cccccc}
\hline $\mathrm{Zn}(1)$ & 2.027 & $\mathrm{Zn}(2)$ & 2.019 & $\mathrm{Nb}(1)$ & 4.968 \\
$\mathrm{Nb}(2)$ & 4.968 & $\mathrm{O}(1)$ & 1.821 & $\mathrm{O}(2)$ & 1.830 \\
$\mathrm{~F}(1)$ & 0.711 & $\mathrm{~F}(2)$ & 0.740 & $\mathrm{~F}(3)$ & 0.756 \\
$\mathrm{~F}(4)$ & 0.749 & $\mathrm{~F}(5)$ & 0.745 & $\mathrm{~F}(6)$ & 0.703 \\
$\mathrm{~F}(7)$ & 0.754 & $\mathrm{~F}(8)$ & 0.715 & $\mathrm{~F}(9)$ & 0.753 \\
$\mathrm{~F}(10)$ & 0.754 & & & & \\
\hline
\end{tabular}


Table S13. Bond valence sum for compound 6

\begin{tabular}{cccccc}
\hline $\mathrm{Cd}(1)$ & 2.292 & $\mathrm{Nb}(1)$ & 5.080 & $\mathrm{O}(1) / \mathrm{F}(1)$ & 1.400 \\
$\mathrm{~F}(2)$ & 0.767 & $\mathrm{~F}(3)$ & 0.760 & & \\
\hline
\end{tabular}

\section{Reasons for alert level B and A}

The alert level $\mathrm{B}$ and $\mathrm{A}$ for compounds 1 and 2 are raised from the incompleteness of the single crystal X-ray diffraction data collection obtained using synchrotron radiation. The complete single crystal X-ray diffraction data set might not be obtained for the all sites of crystal during the data collection using the single axis rotation method on a BL2D-SMC at the Pohang Light Source II. ${ }^{1,2}$

1. Shin, J. W.; Eom, K.; Moon, D. J. J. o. s. r., BL2D-SMC, the supramolecular crystallography beamline at the Pohang Light Source II, Korea. J. Synchrotron Rad. 2016, 23, 369-373.

2. Moon, D.; Choi, J.-H., Crystal structure of ammonium bis (pyridine-2, 6dicarboxylato-k ${ }^{3} \mathrm{O}, \mathrm{N}, \mathrm{O}^{\prime}$ ) chromate (III) from synchrotron data. Acta. Cryst. 2015, E71, 210212. 